\title{
Alteração de sinais vitais e desfecho clínico de pacientes admitidos em unidade de emergência
}

Alteration in vital signs and clinical outcome of patients admitted to an emergency unit

Alteraciones de los signos vitales y resultado clínico de pacientes admitidos en una unidad de emergencias

\section{Gabriella Novelli Oliveira ${ }^{\mathrm{I}}$, Tallita Costa Reis ${ }^{\mathrm{II}}$, Diná de Almeida Lopes Monteiro da Cruz ${ }^{\mathrm{III}}$, Lilia de Souza Nogueira ${ }^{\text {IV }}$}

\begin{abstract}
Resumo: Objetivo: descrever as características dos registros de enfermagem, incluindo os sinais vitais, e comparar o desfecho clínico dos pacientes segundo a presença de alteração dos sinais vitais no ambiente de emergência. Método: estudo transversal, com análise retrospectiva de prontuários de pacientes adultos admitidos em maio/2018 em um Pronto-Socorro de São Paulo. Os dados dos registros de enfermagem coletados foram inseridos no sistema REDCap e análises descritivas e inferenciais foram realizadas. Resultados: dos 194 prontuários (54,1\% masculino, idade média 59,7 anos) a queixa de entrada, comorbidades e primeiras condutas realizadas na emergência foram os registros de enfermagem mais anotados. Frequências cardíaca e respiratória e pressão arterial foram os sinais vitais mais alterados e associados ao óbito. Conclusão: a clareza e a frequência dos registros de enfermagem, assim como a correta interpretação dos sinais vitais, são componentes essenciais para a segurança do cuidado prestado ao paciente na emergência.
\end{abstract}

Descritores: Registros de enfermagem; Cuidados de enfermagem; Sinais vitais; Serviço hospitalar de emergência; Emergências

Abstract: Objective: to describe the characteristics of the nursing records, including the vital signs, and to compare the patient's clinical outcome according to the presence of alterations in the vital signs in the emergency setting. Method: a cross-sectional study, with retrospective analysis of patient records of adult individuals admitted in May 2018 in an Emergency Room of São Paulo. The data collected from the nursing records were inserted in the REDCap system and descriptive and inferential analyses were carried out. Results: of the 194 patient records $(54.1 \%$ male, mean age of 59.7 years old), the complaints at admission, comorbidities and first care measures

\footnotetext{
${ }^{I}$ Enfermeira. Doutoranda pela Escola de Enfermagem da Universidade de São Paulo. Hospital Universitário da Universidade de São Paulo. São Paulo- SP. Brasil. E-mail: gabinovelli@usp.br. Orcid: https://orcid.org/0000-0003-2100-1937

II Enfermeira. Graduada pela Escola de Enfermagem da Universidade de São Paulo. São Paulo- SP. Brasil. E-mail: tallita.reis@usp.br. Orcid: https://orcid.org/0000-0002-0721-2968

III Enfermeira. Professora do Departamento de Enfermagem Médico-Cirúrgica da Escola de Enfermagem da Universidade de São Paulo. São Paulo- SP. Brasil. E-mail: dinamcruz@usp.br. Orcid: https://orcid.org/0000-0003-1373-409X

IV Enfermeira. Professora do Departamento de Enfermagem Médico-Cirúrgica da Escola de Enfermagem da Universidade de São Paulo. São Paulo- SP. Brasil. E-mail: lilianogueira@usp.br. Orcid: https://orcid.org/0000-0001-5387-3807
} 
Alteração de sinais vitais e desfecho clínico de pacientes admitidos em unidade de... | 2

carried out in the emergency room were the most written down records. Heart and respiratory rates and blood pressure were the most altered and death-associated vital signs. Conclusion: the clarity and frequency of the nursing records, as well as the correct interpretation of the vital signs, are essential components for the safety of the care provided to the emergency patient.

Descriptors: Nursing records; Nursing care; Vital signs; Emergency service, hospital; Emergencies

Resumen: Objetivo: describir las características de los registros de Enfermería, incluidos los signos vitales, y comparar el resultado clínico de los pacientes según la presencia de alteraciones de los signos vitales en el entorno del servicio de emergencia. Método: estudio transversal, con análisis retrospectivo de historias clínicas de pacientes adultos admitidos en mayo de 2018 en una Unidad de Emergencias de San Pablo. Los datos de los registros recolectados se cargaron al sistema REDCap ${ }^{\circledR}$ y se realizaron análisis descriptivos e inferenciales. Resultados: en 194 historias clínicas (54,1\% de pacientes masculinos con media de 59,7 años), el motivo de consulta inicial, las comorbilidades y las primeras acciones realizadas en el área de emergencias fueron los registros de enfermería más anotados. La frecuencia cardíaca, el ritmo respiratorio y la presión arterial fueron los signos vitales que presentaron mayores alteraciones y estuvieron más relacionados con un resultado final de fallecimiento. Conclusión: la claridad y la frecuencia de los registros de Enfermería, al igual que la correcta interpretación de los signos vitales, son componentes esenciales para la seguridad en la atención que se brinda a los pacientes en el área de Emergencias.

Descriptores: Registros de enfermería; Atención de enfermería; Signos vitales; Servicio de urgencia en hospital; Urgencias médicas

\section{Introdução}

Os registros clínicos são uma das formas de comunicação escrita da equipe para promover a continuidade da assistência e devem refletir o processo de enfermagem, indicando as decisões fundamentais para o cuidado (os diagnósticos, os resultados e as intervenções). ${ }^{1-3}$ Tais registros contêm informações importantes do atendimento prestado ao paciente e devem ser feitos de forma organizada no prontuário. ${ }^{3-4}$ No contexto da assistência, dados sobre a condição do paciente, a alteração do seu estado clínico, os procedimentos realizados e as condutas tomadas devem ser anotados. ${ }^{1,3}$ Porém, a literatura aponta que há falhas nessas anotações que, muitas vezes, não refletem a realidade da assistência. ${ }^{1-2}$ Diante desse contexto e da importância da qualidade dos registros realizados, o Conselho Federal de Enfermagem (COFEN) aprovou, em 2016, a resolução no 0514 que orienta os profissionais sobre as informações essenciais que devem ser anotadas por meio do manual "Guia de recomendações para registros de enfermagem em prontuário do paciente”. ${ }^{3}$ Recentemente uma pesquisa 
nacional no ambiente de terapia intensiva verificou que a qualidade dos registros é frequentemente observada como inadequada nas determinações legais conforme as orientações do COFEN e que não há clareza na avaliação do estado do paciente. ${ }^{5}$

No ambiente de urgência e emergência hospitalar, ainda são escassos os estudos nacionais que avaliam a qualidade dos registros de enfermagem. ${ }^{2,5} \mathrm{O}$ contexto do pronto-socorro deve ser motivo de investigação dessas anotações, pois é um ambiente complexo e heterogêneo devido aos diferentes níveis de gravidade e tempos de permanência dos pacientes clínicos, cirúrgicos e de trauma. ${ }^{6-7}$ Muitos pacientes ficam internados por mais de vinte e quatro horas nesse setor e a equipe de enfermagem é a que mais presta cuidados diretos a esses doentes e, portanto, a documentação dessa assistência deveria ser fidedigna e completa. ${ }^{6-7}$

O registro da condição clínica, incluindo os sinais vitais, no ambiente de emergência permite que o plano de cuidados seja o mais adequado para a complexidade de cada paciente. ${ }^{6}$ Além disso, os sinais vitais têm sido tratados como indicadores precoces de deterioração do estado dos pacientes hospitalizados, sendo que a interpretação de seus valores precisa ser adequada para refletir o uso de tais informações nos processos decisórios dos enfermeiros. ${ }^{6-7}$

O manejo precoce do paciente que apresenta sinais de piora clínica por meio da avaliação dos sinais vitais pode prevenir ocorrências de eventos adversos como parada cardiorrespiratória (PCR), morte intra-hospitalar e internação não planejada na Unidade de Terapia Intensiva (UTI). ${ }^{6,8}$ Estudo australiano observou que, no ambiente de emergência, os sinais vitais registrados com maior frequência foram pressão arterial, frequência cardíaca e saturação periférica de oxigênio. Frequência respiratória, temperatura e avaliação do estado mental foram os menos registrados. ${ }^{7}$

Sabe-se que a avaliação dos parâmetros vitais ainda é falha, incompleta e sem o registro adequado em enfermarias e no ambiente de emergência. ${ }^{7,9}$ A avaliação sistematizada dos sinais vitais por meio de escalas de detecção precoce é aplicada internacionalmente e tem auxiliado a equipe de saúde na identificação de sinais de agravo clínico e registros mais completos. ${ }^{7,9}$ Esses 
Alteração de sinais vitais e desfecho clínico de pacientes admitidos em unidade de... | 4

dados também podem ser usados para verificar o grau de gravidade e mortalidade dos pacientes na emergência pela escala Rapid Emergency Medicine Score (REMS). ${ }^{8,10-11}$ Portanto, a literatura internacional tem colocado a importância da vigilância dos sinais vitais na identificação precoce de agravo e gravidade do paciente. A enfermagem é fundamental nesse processo, pois é a equipe que realiza o controle dos parâmetros vitais e deve estar apta para reconhecer e agir frente à identificação de qualquer alteração dos mesmos. ${ }^{6-7}$

A mensuração e o registro fidedigno dos parâmetros fisiológicos dos pacientes em unidades de emergência são importantes para garantir cuidado de qualidade e centrado nas necessidades de cada doente. ${ }^{1,69}$ Nesse sentido, o objetivo deste estudo foi descrever as características dos registros de enfermagem, incluindo os sinais vitais, e comparar o desfecho clínico dos pacientes segundo a presença de alteração dos sinais vitais no ambiente de emergência.

\section{Método}

Trata-se de um estudo transversal. A população foi composta por prontuários de pacientes atendidos no período de 01/05/2018 a 31/05/2018 em um Pronto-Socorro Adulto (PSA) público de nível secundário da cidade de São Paulo. O PSA presta atendimento em clínica médica, cirurgia geral e ortopedia, com média de 300 pacientes admitidos por mês.

O setor de observação possui dez leitos com monitor multiparamétrico e rede de oxigênio, além de capacidade para dez macas extras. No PSA é documentado o processo de enfermagem, de forma manual, em prontuário físico para todos os pacientes em observação clínica. O enfermeiro realiza a admissão do paciente, define os diagnósticos, resultados esperados e a prescrição de enfermagem. A cada 24 horas é realizada a evolução do paciente em relação a esses diagnósticos e resultados. Os registros dos cuidados, incluindo os sinais vitais, são realizados pela equipe de enfermagem em impressos manuais próprios chamados "anotação de enfermagem" e "controle de sinais vitais". 
A rotina no setor de observação é o controle dos sinais vitais (temperatura corporal, frequência cardíaca, pressão arterial, frequência respiratória e dor) no início do plantão e a cada seis horas, compatível com o turno de trabalho da equipe de enfermagem. Neste estudo, foram considerados todos os parâmetros registrados a cada seis horas e a ausência de anotação de algum sinal vital no turno analisado foi considerada como não registrado. Para efeito deste estudo, um registro de enfermagem foi definido como o conjunto de todas as anotações feitas no prontuário do paciente a cada seis horas; portanto, cada plantão noturno incluiu dois "registros de enfermagem”, correspondendo a dois períodos de seis horas.

No PSA, a oximetria de pulso é avaliada nos pacientes em observação admitidos em leitos com monitor multiparamétrico. Para os doentes que se encontram em macas extras, esse parâmetro é obtido por oxímetros portáteis; portanto, a avaliação desse parâmetro nos pacientes de maca extra não é uma rotina. A dor é avaliada por meio de escala numérica. Não existe uma padronização quanto à frequência da avaliação do estado mental, sendo a Escala de Coma de Glasgow habitualmente aplicada nos pacientes traumatizados.

Foram considerados os seguintes critérios de inclusão dos prontuários dos pacientes: ter idade maior ou igual a 15 anos e ser admitido no PSA em leito com monitor multiparamétrico ou em maca extra. Foram excluídos os prontuários com ausência de informação de idade, sexo, tipo de alta e/ou letra ilegível. Os prontuários dos pacientes que chegaram no PSA em PCR e não apresentaram retorno da circulação espontânea não foram incluídos na amostra, uma vez que esses casos não são admitidos em leito de observação.

O Serviço de Arquivo Médico e Estatística (SAME) do hospital disponibilizou uma lista com 319 números de prontuários de pacientes que ficaram em observação no PSA no período do estudo. Desses, 87 prontuários estavam guardados em um local externo ao SAME sem possibilidade de disponibilização no período da coleta de dados. Ainda dessa mesma lista, 15 registros de prontuários estavam repetidos e nove eram de pacientes que chegaram em PCR sem 
Alteração de sinais vitais e desfecho clínico de pacientes admitidos em unidade de... | 6

retorno à circulação espontânea. Dos 208 prontuários disponíveis, 14 foram excluídos devido dados incompletos, restando 194 prontuários para análise final.

Desses prontuários, foram coletadas variáveis sociodemográficas (idade, sexo e antecedentes pessoais), do tempo de observação do paciente no PSA (especialidade médica de atendimento, tempo de permanência na unidade e tipo de alta - residência, internação, transferência ou óbito), dos registros de enfermagem (admissão pela enfermeira, relato das condições neurológica, cardiovascular e respiratória do paciente, dispositivos presentes acessos venosos, suporte ventilatório, cateter vesical ou enteral, dreno e imobilizações - data, hora, assinatura, carimbo e sinais vitais). Dos sinais vitais, foram coletadas as frequências de registro da temperatura corporal, frequência cardíaca, pressão arterial média, frequência respiratória, saturação periférica de oxigênio e dor.

Dados do registro sobre as condutas realizadas quando algum desses sinais apresentava alteração foram analisados. A busca de tais registros ocorria quando se identificava algum sinal vital alterado, segundo os parâmetros considerados normais (Quadro 1) pela escala REMS.11-12 A REMS estima a gravidade e prediz a mortalidade do paciente no ambiente de emergência por meio da avaliação de sinais vitais e idade do paciente. Como a temperatura corporal não consta entre os parâmetros da REMS, os autores deste estudo definiram como temperatura alterada quando $>37,8^{\circ} \mathrm{C}$ - conforme rotina da unidade. Como já mencionado, a Escala de Coma de Glasgow não é aplicada em todos os pacientes da unidade, razão pela qual esse parâmetro não foi analisado.

Quadro 1- Parâmetros normais de sinais vitais segundo a escala REMS. São Paulo, SP, Brasil, 2018.

\begin{tabular}{|c|c|c|c|}
\hline $\begin{array}{c}\text { Frequência } \\
\text { cardíaca } \\
(\mathrm{BPM})^{*}\end{array}$ & $\begin{array}{c}\text { Frequência } \\
\text { respiratória } \\
(\mathrm{IRPM})^{\dagger}\end{array}$ & $\begin{array}{c}\text { Pressão arterial } \\
\text { média } \\
(\mathrm{mmHg})^{\ddagger}\end{array}$ & $\begin{array}{c}\text { Saturação periférica de } \\
\text { oxigênio } \\
(\%) \S\end{array}$ \\
\hline $70-109$ & $12-24$ & $70-109$ & $>89$ \\
\hline
\end{tabular}

*BPM-batimentos por minuto; ${ }^{\dagger}$ IRPM-incursões respiratórias por minuto; ${ }^{\ddagger} \mathrm{mmHg}$-milímetros de mercúrio; $\S \%$ - porcentagem.

Fonte: Parâmetros dos sinais vitais da escala REMS, ${ }^{11-12}$ adaptada ao estudo. 
Para a coleta de dados, primeiro houve o treinamento de duas das pesquisadoras para utilizar o sistema Research Electronic Data Capture (REDCap $).{ }^{13}$ As ferramentas utilizadas do REDCap foram: coleta eletrônica, gerenciamento dinâmico e exportação dos dados. Previamente, foi realizado teste piloto com dez prontuários e, na vigência de qualquer divergência durante o teste piloto e coleta de dados, uma terceira pesquisadora era consultada.

Estatísticas descritivas e inferenciais foram realizadas na análise dos dados. Para a análise do desfecho, os pacientes foram divididos em dois grupos: sobreviventes (doentes que receberam alta, foram transferidos ou evadiram do PSA, além dos internados na instituição) ou não sobreviventes (doentes que evoluíram a óbito no PSA). Para a comparação desses grupos segundo sinais vitais alterados, foram utilizados os testes Qui-Quadrado de Pearson, BrunnerMunzel e Exato de Fisher, com nível de significância de 5\%. O programa R versão 3.6.1 foi utilizado para a análise dos dados.

O estudo foi aprovado pelo Comitê de Ética em Pesquisa da Instituição Pública Estadual no dia 06 de julho de 2018 sob parecer nº 2.759.136, de acordo com a Resolução 466/12 do Conselho Nacional de Saúde. Houve dispensa da aplicação do termo de consentimento livre e esclarecido para pacientes/familiares, uma vez que tratava de uma pesquisa retrospectiva de análise de prontuários, sem que houvesse qualquer prejuízo e interferência no atendimento e tratamento do paciente na instituição. As pesquisadoras se comprometeram a garantir o sigilo e confiabilidade dos dados coletados e os prontuários foram consultados com cuidado e responsabilidade a fim de evitar extravios, rasuras e perda do sigilo.

\section{Resultados}

Dos 194 prontuários analisados, 105 (54,1\%) eram de pacientes do sexo masculino, com média de idade de 59,7 anos $(\mathrm{DP} \pm 20,4)$ e variação de 17 a 99 anos. Em relação às comorbidades, 
Alteração de sinais vitais e desfecho clínico de pacientes admitidos em unidade de... $\mid 8$

$89(45,9 \%)$ apresentavam hipertensão arterial sistêmica; 44 (22,7\%) diabetes mellitus; 23 (11,9\%) cardiopatia; 17 (8,8\%) acidente vascular encefálico e 13 (6,7\%) doença oncológica.

Houve predomínio de atendimento da especialidade de clínica médica ( $\mathrm{n}=93 ; 48 \%)$, seguida da cirurgia geral $(\mathrm{n}=79 ; 40,7 \%)$ e ortopedia $(\mathrm{n}=22 ; 11,3 \%)$. O tempo médio de permanência no PSA foi de 1,3 dia com máximo de sete dias. Do total, 72 (37,1\%) pacientes receberam alta hospitalar, 67 (34,5\%) foram encaminhados para unidade de internação, sete $(3,6 \%)$ para UTI, 27 (14\%) transferidos para outro hospital, $18(9,3 \%)$ morreram no PSA durante o período de observação e três $(1,5 \%)$ evadiram.

Quanto aos registros dos enfermeiros na admissão do paciente, verificou-se que em 140 $(72,2 \%)$ prontuários constava o relato do meio de transporte pelo qual o paciente chegou ao serviço, em 192 (99\%) o registro da queixa de entrada, em $190(97,9 \%)$ havia a descrição da condição física do paciente ao chegar no serviço, em $172(88,7 \%)$ o registro das comorbidades do paciente e em $145(74,7 \%)$ constavam as primeiras condutas realizadas no setor.

Tabela 1 - Descrição das categorias de conteúdo do registro de enfermagem. São Paulo, SP, Brasil, 2018.

\begin{tabular}{|c|c|c|c|c|}
\hline \multirow{3}{*}{ Categorias de conteúdo } & \multicolumn{4}{|c|}{ Registro de enfermagem } \\
\hline & \multicolumn{2}{|c|}{ Sim } & \multicolumn{2}{|c|}{ Não } \\
\hline & $\mathrm{n}$ & $\%$ & $\mathrm{n}$ & $\%$ \\
\hline Descrição da condição neurológica & 765 & 95,4 & 37 & 4,6 \\
\hline Descrição dos dispositivos presentes & 773 & 96,4 & 29 & 3,6 \\
\hline Descrição da condição respiratória & 216 & 26,9 & 586 & 73,1 \\
\hline Descrição da condição cardiovascular & 135 & 16,8 & 667 & 83,2 \\
\hline Data & 714 & 89,0 & 88 & 11,0 \\
\hline Hora & 792 & 98,8 & 10 & 1,2 \\
\hline Assinatura do profissional & 738 & 92,0 & 64 & 8,0 \\
\hline Carimbo do profissional & 726 & 90,5 & 76 & 9,5 \\
\hline
\end{tabular}

Nota: $\mathrm{n}=$ total de registros de enfermagem 
A permanência na emergência dos 194 pacientes cujos prontuários foram analisados totalizou 802 turnos de seis horas, o que correspondeu, conforme descrito no método, a 802 registros de enfermagem. Destacam-se, na Tabela 1, que as condições respiratória e cardiovascular dos pacientes foram as menos registradas pela equipe de enfermagem.

Tabela 2 - Registros dos sinais vitais. São Paulo, SP, Brasil, 2018.

\begin{tabular}{|c|c|c|c|c|}
\hline \multirow{3}{*}{ Sinais vitais } & \multicolumn{4}{|c|}{ Registro de enfermagem } \\
\hline & \multicolumn{2}{|c|}{ Sim } & \multicolumn{2}{|c|}{ Não } \\
\hline & $\mathrm{n}$ & $\%$ & $\mathrm{n}$ & $\%$ \\
\hline Pressão arterial média & 797 & 99,4 & 5 & 0,6 \\
\hline Frequência cardíaca & 797 & 99,4 & 5 & 0,6 \\
\hline Frequência respiratória & 794 & 99,0 & 8 & 1,0 \\
\hline Temperatura & 794 & 99,0 & 8 & 1,0 \\
\hline Dor & 706 & 88,0 & 96 & 12,0 \\
\hline Saturação periférica de oxigênio & 400 & 49,9 & 402 & 50,1 \\
\hline
\end{tabular}

Os dados da Tabela 2 retratam a anotação dos sinais vitais referente aos 802 registros de enfermagem. Nota-se que a saturação periférica de oxigênio foi o sinal vital menos registrado. O conjunto dos sinais vitais (pressão arterial média, frequência cardíaca, frequência respiratória, saturação periférica de oxigênio, temperatura e dor) foi verificado uma única vez em 616 (76,8\%) dos 802 registros de enfermagem. 
Alteração de sinais vitais e desfecho clínico de pacientes admitidos em unidade de... $\mid 10$

Tabela 3 - Sinais vitais segundo frequência de valores fora na normalidade. São Paulo, SP, Brasil, 2018.

\begin{tabular}{|c|c|c|c|c|}
\hline \multirow{3}{*}{ Sinais vitais } & \multicolumn{4}{|c|}{ Alterado } \\
\hline & \multicolumn{2}{|c|}{ Sim } & \multicolumn{2}{|c|}{ Não } \\
\hline & $\mathrm{n}$ & $\%$ & $\mathrm{n}$ & $\%$ \\
\hline Pressão arterial média & 186 & 23,3 & 611 & 76,7 \\
\hline Frequência cardíaca & 172 & 21,6 & 625 & 78,4 \\
\hline Frequência respiratória & 75 & 9,4 & 716 & 90,6 \\
\hline Temperatura & 35 & 4,4 & 759 & 95,6 \\
\hline Saturação periférica de oxigênio & 12 & 3,0 & 388 & 97,0 \\
\hline
\end{tabular}

Nota: $\mathrm{n}=$ total dos registros de sinais vitais

Do total de 4.288 sinais vitais registrados foi observado que, em 480 (11,2\%) desses, algum parâmetro estava alterado. A Tabela 3 mostra as frequências de cada sinal vital alterado. Observa-se que a pressão arterial média, a frequência cardíaca e a frequência respiratória foram os parâmetros vitais que apresentaram alterações com maior frequência.

Em 108 (22,5\%) dos 480 registros de sinais vitais alterados houve anotações das condutas realizadas. Suporte ventilatório, como manejo da via aérea, colocação de cateter nasal, máscara ventilatória e ventilação mecânica, foi a conduta mais registrada ( $n=70 ; 64,8 \%$ ), seguida da administração de medicamentos, incluindo droga vasoativa e reposição volêmica (n=26; $24,0 \%$ ) e de outras condutas $(n=12 ; 11,1 \%)$ como jejum, passagem de dreno de tórax e comunicação à equipe médica. 
Tabela 4 - Desfecho clínico dos pacientes no PSA segundo presença de sinal vital alterado. São Paulo, SP, Brasil, 2018.

\begin{tabular}{|c|c|c|c|c|c|c|}
\hline \multirow{3}{*}{ Sinal vital alterado } & \multirow{3}{*}{ Categoria } & \multicolumn{4}{|c|}{ Desfecho clínico no PSA } & \multirow{3}{*}{$\mathrm{p}$} \\
\hline & & \multicolumn{2}{|c|}{ Sobreviventes } & \multicolumn{2}{|c|}{ Não sobreviventes } & \\
\hline & & $\mathrm{n}$ & $\%$ & $\mathbf{n}$ & $\%$ & \\
\hline \multirow{2}{*}{ Frequência cardíaca } & Sim & 60 & 82,2 & 13 & 17,8 & \multirow{2}{*}{$0,002^{*}$} \\
\hline & Não & 116 & 95,9 & 5 & 4,1 & \\
\hline \multirow{2}{*}{ Frequência respiratória } & Sim & 21 & 72,4 & 8 & 27,6 & \multirow{2}{*}{$<0,001^{*}$} \\
\hline & Não & 155 & 93,9 & 10 & 6,1 & \\
\hline \multirow[b]{2}{*}{ Saturação periférica de oxigênio } & Sim & 5 & 62,5 & 3 & 37,5 & \multirow[b]{2}{*}{$0,028^{+}$} \\
\hline & Não & 171 & 91,9 & 15 & 8,1 & \\
\hline \multirow{2}{*}{ Pressão arterial média } & Sim & 67 & 81,7 & 15 & 18,3 & \multirow{2}{*}{$<0,001^{*}$} \\
\hline & Não & 109 & 97,3 & 3 & 2,7 & \\
\hline \multirow{2}{*}{ Temperatura } & Sim & 6 & 60,0 & 4 & 40,0 & \multirow{2}{*}{$0,008^{+}$} \\
\hline & Não & 170 & 92,4 & 14 & 7,6 & \\
\hline
\end{tabular}

Nota: $\mathrm{n}=$ total de pacientes; ${ }^{*}$ Teste Qui Quadrado de Pearson; ${ }^{\dagger}$ Teste Exato de Fisher

Dos 194 pacientes, $122(62,8 \%)$ tiveram algum sinal vital alterado, sendo que foi apresentado, em média, 1,2 sinal vital alterado no período de permanência no PSA. Segundo o teste Brunner-Munzel, houve diferença significativa $(\mathrm{p}<0,001)$ da média do número de sinais vitais alterados entre os sobreviventes $(1,0 \pm 1,1)$ e os não sobreviventes $(2,6 \pm 1,5)$. Na comparação dos grupos (sobreviventes e não sobreviventes), observa-se que houve diferença significativa entre eles em relação à presença de alteração de todos os sinais vitais analisados, com maior probabilidade de óbito entre os pacientes que apresentaram alguma alteração durante a permanência no PSA (Tabela 4).

\section{Discussão}

Os resultados deste estudo mostraram as principais características da documentação de enfermagem no ambiente de emergência, sendo possível observar que os registros da frequência 
Alteração de sinais vitais e desfecho clínico de pacientes admitidos em unidade de... $\mid 12$

cardíaca, pressão arterial, frequência respiratória e temperatura foram completos na maioria da amostra. O registro de sinal vital alterado esteve presente em $62,8 \%$ da amostra, fato que coloca em discussão a importância do controle rigoroso dos sinais vitais no ambiente de emergência. Porém, na documentação das condutas de enfermagem frente aos parâmetros fisiológicos alterados, foi verificado que em apenas $22,8 \%$ dos prontuários tinha uma adequada anotação das condutas tomadas.

$\mathrm{Na}$ documentação de enfermagem da admissão, a maioria dos registros foi sobre a condição física do paciente, assim como o meio pelo qual ele chegou à unidade, a queixa principal, as comorbidades presentes e as condutas iniciais. Para o enfermeiro da unidade de emergência, a avaliação com informações sobre a condição física e queixa inicial do doente no contexto do processo de enfermagem oferece subsídios necessários para traçar o plano de cuidados segundo as prioridades diagnósticas encontradas, possibilitando as intervenções de enfermagem adequadas para cada doente..$^{4,7}$

Assim como em outros estudos nacionais, ${ }^{1,4}$ houve uma pequena porcentagem de falta de informação da data (11\%) e carimbo do profissional $(9,5 \%)$ nesta pesquisa. A literatura aponta que a ausência de data, hora e carimbo, itens considerados necessários para um adequado registro de enfermagem segundo o COFEN, é um comportamento constante no cotidiano da enfermagem que pode comprometer o processo avaliativo e de auditoria dos prontuários. ${ }^{1,4,14} \mathrm{~A}$ documentação eletrônica no ambiente de emergência pode contribuir para reduzir parte desse problema, além de dar suporte para a coleta e armazenamento dos dados, raciocínio clínico e padronização da linguagem. ${ }^{2}$

Os resultados deste estudo confirmam que os registros dos sinais vitais são fundamentais no ambiente hospitalar, inclusive no Pronto-Socorro. É de responsabilidade da equipe de enfermagem a mensuração dos sinais vitais na constante vigilância para identificar e interpretar anormalidades dos parâmetros fisiológicos que pode sinalizar piora da condição clínica. ${ }^{6,15}$ Nos hospitais da Inglaterra que compõem o sistema National Health Service (NHS), o intervalo 
máximo recomendado para controle dos sinais vitais é de 12 horas; já na Austrália, conforme as orientações do The Australian National Consensus Statement on recognising and responding to clinical deterioration, esse intervalo é de 8 horas e os sinais vitais controlados são pressão arterial, frequência cardíaca, frequência respiratória, saturação periférica de oxigênio, temperatura e nível de consciência. ${ }^{9,15}$ Neste estudo, foi observado que o intervalo de controle de sinais foi de 6 horas na maioria da amostra, conforme a rotina da unidade investigada. No Brasil, ainda não existe um consenso estabelecido do intervalo do controle de sinais vitais no ambiente hospitalar e o nível de consciência ainda não está incorporado rotineiramente na avaliação do controle dos sinais vitais. ${ }^{3}$

Quando avaliados os registros dos sinais vitais, dados sobre frequência cardíaca, temperatura, frequência respiratória e pressão arterial média foram os mais completos. Resultado semelhante aconteceu em estudo australiano que avaliou os registros dos sinais vitais no ambiente de emergência e enfermaria de clínica médica e cirúrgica. ${ }^{7}$

A documentação da saturação periférica de oxigênio foi observada em apenas 49,9\% da amostra. Em contrapartida, estudo australiano verificou que no ambiente de emergência a saturação de oxigênio foi um dos parâmetros mais avaliados e registrados. ${ }^{7}$ Como já mencionado anteriormente, a avaliação desse parâmetro é realizada nos pacientes que permanecem em observação nos leitos com monitor multiparamétrico do PSA. É possível observar que, apesar dos poucos registros da condição respiratória na casuística deste estudo, a anotação da frequência respiratória foi satisfatória sendo importante destacar que esse sinal é um parâmetro considerado indicador de gravidade, por isso a necessidade de uma avaliação e registro adequado no ambiente hospitalar..$^{7-8}$

Apesar da avaliação do nível de consciência ainda não ser considerada rotina do controle dos sinais vitais no contexto brasileiro, a avaliação sistemática dos parâmetros fisiológicos, 
Alteração de sinais vitais e desfecho clínico de pacientes admitidos em unidade de... | 14

incluindo o nível de consciência por meio de um sistema de detecção precoce, pode auxiliar na identificação de agravos com alta mortalidade, como a sepse..$^{3,16}$

Uma investigação internacional que buscou analisar os critérios do The quick Sequential Organ Failure Assessment (qSOFA) e os sinais vitais utilizados na escala National Early Warning Score (NEWS) em pacientes de emergência na capacidade de predizer admissão em leito de terapia intensiva mostrou que o escore NEWS apresentou comportamento superior ao qSOFA, pois abrange a avaliação completa desses parâmetros, sendo a avaliação do nível de consciência um dos fatores discutidos no estudo para o desempenho melhor desse escore em relação ao qSOFA. ${ }^{16}$

A adoção de uma escala de detecção precoce de gravidade que inclua a avaliação do nível de consciência na rotina de controle de sinais vitais pode ser interessante na realidade brasileira. Na Inglaterra, os hospitais do NHS já utilizam a NEWS, um sistema de detecção precoce de gravidade, e relatam que tal prática permitiu uma padronização da linguagem e uma avaliação sistematizada com medidas frequentes dos sinais vitais. ${ }^{17}$

A pressão arterial e as frequências cardíaca e respiratória foram os sinais vitais com maior frequência de alterações. Achado semelhante foi identificado em estudo australiano que observou a frequência dos sinais precoces de piora clínica por meio da avaliação dos parâmetros fisiológicos no contexto de emergência. Verificou-se que $1.071(19,6 \%)$ eram medidas alteradas (31\% da pressão arterial sistólica e $20,8 \%$ da frequência cardíaca). ${ }^{15}$ Ressalta-se que as frequências cardíaca e respiratória e a pressão arterial alteradas estão relacionadas à elevada chance de ocorrência de eventos adversos (morte intra-hospitalar, PCR e internação não planejada em leito de terapia intensiva), ${ }^{8,15}$ sendo importante notar que já existe alteração dos sinais vitais em até 48 horas antes que um evento adverso aconteça. ${ }^{8}$

Os pacientes que foram a óbito neste estudo tinham mais que o dobro de sinais vitais alterados comparados àqueles que sobreviveram. As alterações na frequência cardíaca, respiratória, pressão arterial, temperatura e saturação periférica de oxigênio foram associadas 
ao evento do óbito. Esses achados convergem para outra pesquisa que avaliou o conjunto de sinais vitais alterados e os desfechos dos pacientes em um serviço de emergência e observou que, na admissão, a maioria dos homens com idade igual ou superior a 60 anos tinha alteração da frequência cardíaca, respiratória e temperatura. ${ }^{18}$ Ainda nesse estudo, os pacientes que foram a óbito apresentavam mais de dois sinais alterados durante a permanência na emergência. ${ }^{18}$

Portanto, pesquisas reforçam a importância da vigilância rigorosa do conjunto de parâmetros fisiológicos em uma unidade de emergência. ${ }^{6,15,18-19}$ Embora não tenha sido objetivo desta pesquisa verificar as condições dos sinais vitais na alta hospitalar do paciente, chama atenção uma pesquisa americana que coloca em discussão os valores desses sinais no momento da alta e na readmissão hospitalar em sete dias. Pacientes que receberam alta com alteração da frequência cardíaca e respiratória tiveram mais chances de readmissão em sete dias (odds ratio $=1,87$ ) quando comparado à alta hospitalar de pacientes com apenas a alteração de um desses sinais (odds ratio $=1,21) .{ }^{19}$

Outro destaque desta pesquisa foi a baixa frequência das anotações de conduta frente às alterações dos sinais vitais, sendo que os registros de suporte ventilatório e administração de medicamento, medidas fundamentais para permeabilidade da via área e manutenção da circulação, foram os mais encontrados. Os achados dos resultados desta investigação buscam refletir o papel da enfermagem no reconhecimento de alterações dos sinais vitais e o registro adequado, visto que é a profissão que mais passa tempo com o paciente. ${ }^{6,15}$ As omissões dos registros das alterações dos sinais vitais associadas a longas horas de trabalho e, por vezes, o número insuficiente de profissionais da enfermagem podem comprometer a qualidade da assistência e a ocorrência de evento adverso, mesmo não tendo nenhuma evidência que comprove que as longas jornadas de trabalho da enfermagem sejam capazes de trazer menos segurança para o paciente..$^{20-21}$ 
Alteração de sinais vitais e desfecho clínico de pacientes admitidos em unidade de... $\mid 16$

Por fim, a realização da coleta de dados em uma única instituição por meio da análise retrospectiva de prontuários é uma limitação do estudo e tal fato deve ser considerado na generalização dos resultados.

\section{Conclusão}

Os registros de enfermagem no ambiente de emergência tiveram como características principais a descrição da queixa de entrada, condição física e comorbidades dos pacientes, além das primeiras condutas realizadas. A anotação dos sinais vitais foi completa na maioria dos prontuários, sendo a frequência cardíaca, a pressão arterial e a frequência respiratória os parâmetros mais documentados e frequentemente alterados e presentes nos pacientes que foram a óbito. Os pacientes sobreviventes tiveram menos sinais vitais alterados.

Apesar da baixa frequência de registros sobre as condutas tomadas diante dos parâmetros alterados, foram mais anotados o suporte ventilatório e a administração de medicamentos.

A clareza das anotações de enfermagem assim como a correta interpretação dos sinais vitais pode favorecer para uma assistência de enfermagem segura. A frequência do controle dos parâmetros fisiológicos dos pacientes na emergência ainda não é consenso no Brasil. Logo, o uso de escala de detecção precoce de gravidade pode auxiliar na orientação dos profissionais quanto à frequência ideal de mensuração dos sinais vitais e deve ser considerada na prática clínica da emergência.

\section{Referências}

1. Araujo MM, Diniz SOS, Silva PS. Registros de Enfermagem: reflexões sobre o cotidiano do cuidar. ABCS Health Sci. 2017;42(3):161-5. doi: 10.7322/abcshs.v42i3.920

2. Paese F, Sassol GTMD, Collal GW. Metodologia de estruturação do processo de Enfermagem informatizado para as Unidades de Emergência. Rev Bras Enferm. 2018;71(3):1079-84. doi: 10.1590/00347167-2016-0619

3. BRASIL. Conselho Federal de Enfermagem. Resolução Cofen № 0514/2016. Brasília, DF: Conselho Rev. Enferm. UFSM, Santa Maria, v10, p. 1-19, 2020 
Federal de Enfermagem, 2016. Disponível em: http://www.cofen.gov.br/resolucao-cofen-no05142016_41295.html. Acesso em: 20 fev. 2019.

4. Caldeira MM, Souza TV, Morais RCM, Moraes JRMM, Nascimento LCN, Oliveira ICS. Anotações da equipe de enfermagem: a (des)valorização do cuidado pelas informações fornecidas. Rev Pesq Cuid Fundam. 2019;11(1):135-41. doi: 10.9789/2175-5361.2019.vllil.135-141

5. Aquino MJN, Cavalcante TMC, Abreu RNDC, Scopacasa LF, Negreiros FDS. Anotação de enfermagem: avaliação da qualidade em uma unidade de terapia intensiva. Enferm Foco. 2018;9(1): 07-12. Disponível em: http://revista.cofen.gov.br/index.php/enfermagem/article/view/1314/419

6. Johnson KD, Mueller L, Winkelman C. The nurse response to abnormal vital sign recording in the emergency department. J Clin Nurs. 2016;26:148-56. doi: 10.1111/jocn.13425

7. Considine J, Trotter C, Currey J. Nurse documentations of physiological observations in three acute care setting. J Clin Nurs. 2015;25:134-43. doi: 10.1111/jocn.13010

8. Alam N, Hobbelink EL, Van Tienhoven AJ, Van de Ven PM, Jansma EP, Nanayakkara PWB. The impact of the use of the Early Warning Score (EWS) on patient outcomes: a systematic review. Ressuscitation. 2014;85(5):587-94. doi: 10.1016/j.resuscitation.2014.01.013

9. Smith GB, Recio-Saucedo A, Griffiths P. The measurement frequency and completeness of vital signs in general hospital wards: an evidence free zone? Int J Nurs Stud. 2017;74:A1-4. doi: 10.1016/j.ijnurstu.2017.07.001

10. Hung SK, $\mathrm{Ng}$ CJ, Kuo CF, Goh ZNL, Huang LH, Li CH, et al. Comparison of the mortality in emergency department sepsis score, modified early warning score, rapid emergency medicine score and rapid acute physiology score for predicting the outcomes of adult splenic abscess patients in the emergency department. PLoS One. 2017;12(11):e0187495. doi: 10.1371/journal.pone.0187495

11. Olsson T, Terent A, Lind L. Rapid Emergency Medicine score: a new prognostic tool for in-hospital mortality in nonsurgical emergency department patients. J Intern Med.2004;255(5):579-587. doi:10.1111/j.1365-2796.2004.01321

12. Gök RGY, Gök A, Bulut M. Assessing prognosis with modified early warning score, rapid emergency medicine score and worthing physiological scoring system in patients admitted to intensive care unit from emergency department. Int Emerg Nurs. 2019;43:9-14. doi: 10.1016/j.ienj.2018.06.002

13. Harris PA, Taylor R, Thielke R, Payne J, Gonzalez N, Conde JG. Research electronic data capture (REDCap) - A metadata-driven methodology and workflow process for providing translational research informatics support. J Biomed Inform. 2009;42(2):377-81.

14. Barreto JA, Lima GG, Xavier CF. Inconsistência das anotações de enfermagem no processo de auditoria. R Enferm Cent Oeste Min. 2016;1(6):2081-93. doi: 10.19175/recom.v0i0.917 
Alteração de sinais vitais e desfecho clínico de pacientes admitidos em unidade de... $\mid 18$

15. Lambe K, Currey J, Considine J. Frequency of vital sign assessment and clinical deterioration in an Australian emergency department. Australas Emerg Care. 2016;19(4):217-22. doi: 10.1016/j.aenj.2016.09.001

16. Goulden R, Hoyle MC, Monis J, Railton D, Riley V, Martin P, et al. qSOFA, SIRS and NEWS for predicting inhospital mortality and ICU admission in emergency admissions treated as sepsis. Emerg Med J. 2018;35(6):345-9. doi: 10.1136/emermed-2017-207120

17. Royal College of Physicians (UK). National Early Warning Score (NEWS) 2 [Internet]. 2017 [cited 2018 Nov 18]. Available from: https://www.rcplondon.ac.uk/projects/outputs/national-early-warning-scorenews-2

18. Lee $M$, Taylor $\mathrm{DMcD}$, Ugoni $\mathrm{A}$. The association between abnormal vital groups and undesirable patient outcome. Hong Kong J Emerg Med. 2018;25(3):37-45. doi: 10.1177/1024907917752959

19. Pittappilly M, Sarao MS, Bambach WL, Helmuth A, Nookala V. Vital signs on hospital discharge and re admission rates. QJM. 2019;112(4):275-9. doi: 10.1093/qjmed/hcz002

20. Griffiths P, Ball J, Bloor K, Böhning D, Briggs J, Dall'Ora C, et al. Nurse staffing levels, missed vital signs and mortality in hospitals: retrospective longitudinal observational study. Health Serv Deliv Res. 2018;6(38):1-120. doi: 10.3310/hsdr06380

21. Dall'Ora C, Griffths P, Redfern O, Recio-Saucedo A, Meredith P, Ball J. Nurses' 12-hour shifts and missed or delayed vital signs observations on hospital wards: retrospective observational study. BMJ Open. 2018;9(1):e024778. doi: 10.1136/bmjopen-2018-024778

Editor Científico: Tânia Solange Bosi de Souza Magnago

Editor Associado: Silvana Bastos Cogo

\section{Autor correspondente}

Gabriella Novelli Oliveira

E-mail: gabinovelli@usp.br

Endereço: Av. Prof. Lineu Prestes, 2565 - Butantã, São Paulo- SP

CEP: 05508-000

\section{Contribuições de Autoria}

1 - Gabriella Novelli Oliveira

Concepção do projeto, análise e interpretação dos dados e redação do artigo.

\section{2 - Tallita Costa Reis}


19 | Oliveira GN, Reis TC, Cruz DALM, Nogueira LS

Concepção do projeto, análise e interpretação dos dados e redação do artigo.

\section{3 - Diná de Almeida Lopes Monteiro da Cruz}

Concepção do projeto, revisão crítica relevante do conteúdo intelectual e aprovação final do artigo.

\section{4 - Lilia de Souza Nogueira}

Concepção do projeto, revisão crítica relevante do conteúdo intelectual e aprovação final do artigo.

\section{Como citar este artigo}

Oliveira GN, Reis TC, Cruz DALM, Nogueira LS. Alteração de sinais vitais e desfecho clínico de pacientes admitidos em unidade de emergência. Rev. Enferm. UFSM. 2020 [Acesso em: Anos Mês Dia]; vol.10 e81: 1-19. DOI:https://doi.org/10.5902/2179769242559 